\title{
Simple and fast method for step size determination in computations of signal propagation through nonlinear fibres
}

\section{Rasmussen, Christian Jørgen}

Published in:

Proceedings on Optical Fiber Communication Conference and Exhibit

Link to article, DOI:

10.1109/OFC.2001.928479

Publication date:

2001

Document Version

Publisher's PDF, also known as Version of record

Link back to DTU Orbit

Citation (APA):

Rasmussen, C. J. (2001). Simple and fast method for step size determination in computations of signal propagation through nonlinear fibres. In Proceedings on Optical Fiber Communication Conference and Exhibit (Vol. 3) https://doi.org/10.1109/OFC.2001.928479

\section{General rights}

Copyright and moral rights for the publications made accessible in the public portal are retained by the authors and/or other copyright owners and it is a condition of accessing publications that users recognise and abide by the legal requirements associated with these rights.

- Users may download and print one copy of any publication from the public portal for the purpose of private study or research.

- You may not further distribute the material or use it for any profit-making activity or commercial gain

- You may freely distribute the URL identifying the publication in the public portal 


\title{
Simple and fast method for step size determination in computations of signal propagation through nonlinear fibres
}

\author{
Christian J. Rasmussen \\ Research Center COM, Technical University of Denmark, DK-2800 Lyngby, Denmark \\ phone +45 45256352,fax +4545936581, cr@com.dtu.dk
}

\begin{abstract}
This paper presents a simple and fast method for determination of the step size that exactly leads to a prescribed accuracy when signal propagation through nonlinear optical fibres is computed using the split-step Fourier method.

(C)2000 Optical Society of America

OCIS codes: $(000.4430)$ Numerical approximation and analysis; $(060.4370)$ Nonlinear optics, fibers; $(060.4510)$ Optical

Communications; (060.5530) Pulse propagation and solitons
\end{abstract}

\section{Introduction}

Hundreds of engineers have spent thousands of hours on simulations of optical transmission systems. Therefore it is astonishing that so little has been published about how to compute the signal propagation through nonlinear fibres faster since this is normally the most time consuming part of transmission simulations. It can take days to compute the propagation of WDM signals. The standard method for the propagation computation is the split-step Fourier method whose speed and accuracy. are controlled by the step size. Both computation time and accuracy increase as the step size is reduced so the largest step size compatible with the required accuracy should be chosen to get the best simulation effectiveness. Without a good step size determination method, the employed step size can only be an educated guess of the user making the simulations. To be on the safe side, the chosen step size will typically be far too small which makes the computation time far too long. A step size 4 times smaller than necessary would result in a computation time 4 times longer than necessary, for instance. Add to this that there is still a risk that the educated guess is wrong and that the step size should actually be even smaller to get the required accuracy. For these reasons a reliable step size determination method as the one presented here will improve the simulation effectiveness significantly. What distinguishes this new method from existing ones [1-3] is that it makes it possible to determine the step size that just results in a prescribed accuracy of the computed fibre output signal. The author is not aware of any other method that is capable of this. Moreover, the new method is both simple and general.'

\section{Method for step size determination}

In most system simulations the optical fields in the fibres are represented by scalar complex signals (or envelopes) which obey the nonlinear Schrödinger equation [4]. Thus the computation of the field propagation is reduced to the computation of a fibre output signal, $a_{\text {out exact }}(t)$, from a fibre input signal, $a_{i n}(t)$. The standard numerical method for this is the split-step Fourier method which is considered here in the commonly used symmetrized, constant step size version [4]. The basic principle of the split-step Fourier method is to divide the fibre into sections with a length called the step size, and then let the dispersive and nonlinear effects in each section act on the field alternately. However, letting dispersive and nonlinear effects act alternately rather than at the same time is an approximation so the output signal found using the split-step Fourier method with some step size $h, a_{\text {out,num }}(h ; t)$, deviates from $a_{\text {out,exact }}(t)$. The normalized root-mean-square error $E(h)$ is introduced to quantify this error as a single number

$$
E(h)=\sqrt{\int_{T}\left|a_{\text {out }, \text { num }}(h ; t)-a_{\text {out exact }}(t)\right|^{2} d t / \int_{T}\left|a_{\text {out ,exact }}(t)\right|^{2} d t}
$$

where $T$ is the period of the signals which are assumed to be periodic; a widely used precondition in transmission simulations. In outline $E(h)$ decreases as $h$ tends to 0 , and for sufficiently small $h$ we normally have $E(h) \propto h^{2}$. As an example $E(h)$ is shown on fig. 1 for a WDM propagation case which will be considered more closely below.

An obvious method for determination of the step size $h_{\text {target }}$ which just results in the highest acceptable error $E_{\text {target }}$ is to propagate the field using various step sizes to solve the equation $E\left(h_{\text {target }}\right)=E_{\text {target }}$ numerically. This includes computations that use step sizes much'smaller than $h_{\text {target }}$ in order to approximate $a_{\text {out } \text { exact }}(t)$ in eq. (1). Unfortunately this method is extremely inefficient in its most straightforward implementation because the time spent on the useful computation of the field with step size $h_{\text {target }}$ is only a small fraction of the overhead time spent on the determination of $h_{\text {target }}$. To overcome this problem we propose to compute $E(h)$ on the basis of a properly chosen 


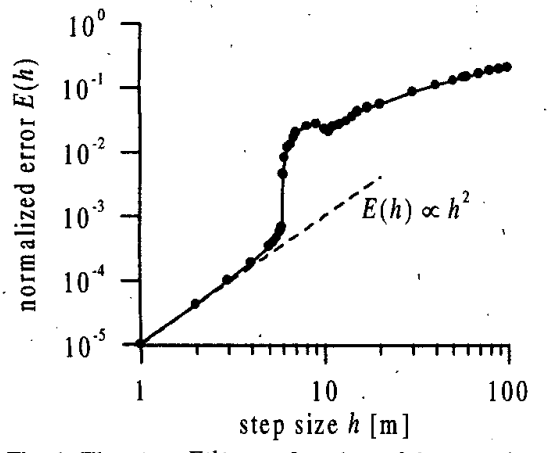

Fig. 1. The error $E(h)$ as a function of the step size $h$ in WDM case v (cf. table 1).

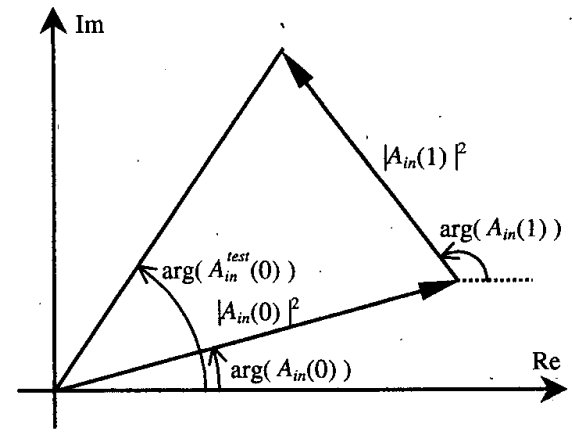

Fig. 2. Construction of the phase of test signal Fourier coefficient $A_{i n}^{\text {test }}(0)$ for $N_{\text {red }}=2$.

test signal $a_{i n}^{t e s t}(t)$ which can be split-stepped through the fibre much faster than the full signal $a_{i n}(t)$. This speed increase is obtained by reducing the number of samples which are used to represent the signals in the computations.

To explain how the test signal is constructed, it is necessary to take a look at the signal representation first. The full signal $a_{i n}(t)$ is represented by $N$ samples taken at time $t=n \cdot T / N, n=0,1 \ldots N-1$ where $T$ is still the period of the full signals. Is it assumed that $a_{i n}(t)$ is band limited to a frequency of $0.5 \cdot N / T \mathrm{~Hz}$, the Fourier coefficients $A_{\text {in }}(m)$ of $a_{\text {in }}(t)$ can be found from the $N$ time samples, and only the $N$ Fourier coefficients $A_{\text {in }}(m), m=-N / 2,-N / 2+1, \ldots, N / 2-1$ can be non-zero. So $a_{i n}(t)$ can be represented by either $N$ time samples or $N$ Fourier coefficients. The test signal represented by only $N / N_{\text {red }}$ samples is now constructed in the frequency domain by defining a signal with period $T / N_{\text {red }}$ and the following Fourier coefficients for $m=-N /\left(2 N_{\text {red }}\right),-N /\left(2 N_{\text {red }}\right)+1, \ldots, N /\left(2 N_{\text {red }}\right)-1$; all other Fourier coefficients are 0 :

$$
\left.\left|A_{i n}^{\text {eest }}(m)\right|=\sqrt{\sum_{i=m \cdot N_{r d}}^{m \cdot N_{r e d}+N_{r e d}-1}\left|A_{i n}(i)\right|^{2}} \quad \arg \left(A_{i n}^{\text {test }}(m)\right)=\arg \left(\sum_{i=m \cdot N_{r e d}}^{m \cdot N_{r e d}+N_{r d}-1}\left|A_{i n}(i)\right| \cdot A_{i n}(i)\right)\right)
$$

According to eq. (2) the power of a frequency component in the test signal equals the total power of the $N_{\text {red }}$ frequency components in the full signal which it represents. The phase of a frequency component in the test signal is a weighted average of the phases of the corresponding $N_{\text {red }}$ frequency components in the full signal as illustrated in fig. 2. It is noted that the test signal has the same spectral width as the full signal since both the number of Fourier. coefficients and the period is reduced with the same factor. As a consequence of the reduced number of samples, a split-step computation on the test signal can be much faster than the corresponding computation on the full signal. For example, using $N_{\text {red }}=64$ as in the examples below, the computation time is reduced by a factor greater than 64 . One reason why the speed is increased more than a factor $N_{\text {red }}$ is that the time it takes to make the Fast Fourier Transforms used in the split-step Fourier method scales as $N \log _{2} N$. Another reason is that a greater part of the data arrays holding the signal samples can be stored in the cache of the computer when the number of samples is reduced.

The step size determination method can now be explained in its entirety. First the test signal is constructed as described above. It is then split-stepped through the fibre using an initial guess step size $h_{\text {ini }}=1000 \mathrm{~m}$, and the computed $a_{\text {out }, \text { inum }}^{\text {test }}\left(h_{\text {ini }} ; t\right)$ is used in eq. (1) to find $E\left(h_{i n i}\right)$. If $E\left(h_{i n i}\right)>E_{\text {target }}$, the step size is halved and the split-stepping is repeated one or more times until $E(h) \leq E_{\text {targer }}$. If $E\left(h_{\text {ini }}\right) \leq E_{\text {target, }}$, the step size is doubled until $E(h)>E_{\text {target }}$. The solution to the equation $E(h)=E_{\text {target }}$ is now bracketed, and $h_{\text {target }}$ is found using a bracketing algorithm such as Ridders' method [5] which refines the step size until $E(h)$ deviates less than $5 \%$ from $E_{\text {target }}$. In the course of these computations, the signal $a_{\text {out, exact }}^{\text {test }}(t)$ in eq. (1) is approximated with $a_{\text {out,num }}^{\text {test }}\left(h_{\text {ref }}, t\right)$ where $h_{\text {ref }}$ is 5 times smaller than the so far smallest step size for which $E(h)$ has been estimated.

It should be emphasized that the proposed step size determination method is general. It is expected to be usable for any numerical fibre model which returns an output signal when it is given an input signal and a precision controlling parameter. This means that the fibre does not have to be described by a particular equation, the numerical method does not have to be the split-step Fourier method, and the precision controlling parameter does not have to be the step size. Furthermore, no assumptions are made as to how $E(h)$ depends on $h$.

\section{Performance of the step size determination method}

An investigation of the performance of the step size determination method must consider both computation time and accuracy. Concerning the latter, there is from the outset no guarantee that $E(h)$ found on the basis of the test signal is a good approximation to $E(h)$ for the full signal. The quality of the approximation depends among other things on the value of $N_{r e d}$, and it is presumed to become better when $N_{\text {red }}$ is decreased since the test signal will then resemble 
the full signal more. But decreasing $N_{\text {red }}$ also means slowing down the computation. A good trade-of between speed and accuracy seems to be $N_{\text {red }}=64$ which is used below. However, the optimum $N_{\text {red }}$ deserves more investigation.

In order to study the performance of the step size determination method, the signal propagation was computed for the five cases defined in table 1. They include transmission of single channel and WDM signals with channel rates of 10 and $40 \mathrm{Gbit} / \mathrm{s}$ through standard single-mode fibre (SMF), dispersion shifted fibre (DSF) and non-zero dispersion shifted fibre (NZDSF).

Table 1. The studied signal propagation cases. (CNRZ: chirped non-return to zero)

\begin{tabular}{|c|c|c|c|c|c|c|c|c|c|}
\hline case & signal & $\begin{array}{l}\text { fibre } \\
\text { type }\end{array}$ & $\begin{array}{l}\text { fibre } \\
\text { length }\end{array}$ & $\begin{array}{l}\text { channel } \\
\text { power }\end{array}$ & $\begin{array}{l}\text { channel } \\
\text { spacing }\end{array}$ & $\begin{array}{c}\text { dispersion at } \\
\text { outermost channels }\end{array}$ & $\begin{array}{l}\text { dispersion } \\
\text { slope }\end{array}$ & $\begin{array}{c}\text { attenua- } \\
\text { tion }\end{array}$ & $\begin{array}{l}\text { nonlinear } \\
\text { coeff. }\end{array}$ \\
\hline $\mathrm{i}$ & $10 \mathrm{Gbit} / \mathrm{s}$ CNRZ & SMF & 250 & 17 & - & 16.8 & 0.07 & 0.2 & 1.3 \\
\hline iii & $40 \mathrm{Gbit} / \mathrm{s} \mathrm{RZ}$ & NZDSF & 150 & 10 & - & 2.3 & 0.07 & 0.22 & 2.16 \\
\hline iv & $16 \times 10 \mathrm{Gbit} / \mathrm{s} \mathrm{NRZ}$ & SMF & 50 & 0 & 100 & $15.52 / 16.48$ & 0.08 & 0.2 & 1.3 \\
\hline$v$ & $64 \times 10 \mathrm{Gbit} / \mathrm{s} \mathrm{NRZ}$ & NZDSF & 80 & 1 & 50 & $1.69 / 3.38$ & 0.067 & 0.2 & 2.0 \\
\hline
\end{tabular}

Table 2 summarizes the performance of the step size determination method in the five cases by listing four performance describing quantities: the step size chosen by the step size determination method $\left(h_{\text {target }}\right)$, the actual normalized root-mean-square error which arises when the full signal is propagated with $h_{\text {target }}\left(E\left(h_{\text {target }}\right)\right)$, the total computation time (step size determination + propagation of full signal) in seconds (time) and the fraction of the total computation time which is spent on finding $h_{\text {target }}(\operatorname{step} \%)$. The quantities are listed for $E_{\text {target }}=10^{-2}, 10^{-4}$ and $10^{-6}$.

Table 2. Performance of the step size determination method. The columns are explained in the text.

\begin{tabular}{|c|c|c|c|c|c|c|c|c|c|c|c|c|}
\hline case & \multicolumn{4}{|c|}{$E_{\text {target }}=10^{-2}$} & \multicolumn{4}{c|}{$E_{\text {target }}=10^{-4}$} & \multicolumn{4}{c|}{$E_{\text {target }}=10^{-6}$} \\
\hline & $h_{\text {target }}[\mathrm{m}]$ & $E\left(h_{\text {target }}\right)$ & time $[\mathrm{s}]$ & step\% & $h_{\text {target }}[\mathrm{m}]$ & $E\left(h_{\text {target }}\right)$ & time $[\mathrm{s}]$ & step\% & $h_{\text {target }}[\mathrm{m}]$ & $E\left(h_{\text {target }}\right)$ & time $[\mathrm{s}]$ & step\% \\
\hline i & 6494 & $1.0 \cdot 10^{-2}$ & 0.3 & 39 & 652 & $1.1 \cdot 10^{-4}$ & 2.2 & 19 & 65.6 & $1.1 \cdot 10^{-6}$ & 21.0 & 18 \\
\hline ii & 16609 & $1.3 \cdot 10^{-2}$ & 0.1 & 36 & 1605 & $1.3 \cdot 10^{-4}$ & 0.3 & 14 & 160 & $1.3 \cdot 10^{-6}$ & 2.6 & 20 \\
\hline iii & 1608 & $1.1 \cdot 10^{-2}$ & 2.2 & 15 & 183 & $1.2 \cdot 10^{-4}$ & 20 & 21 & 18.3 & $1.2 \cdot 10^{-6}$ & 197 & 18 \\
\hline iv & 46.9 & $1.2 \cdot 10^{-2}$ & 77 & 20 & 5.75 & $1.2 \cdot 10^{-4}$ & 582 & 16 & 0.65 & $1.2 \cdot 10^{-6}$ & 5111 & 15 \\
\hline v & 6.24 & $0.92 \cdot 10^{-2}$ & 2925 & 19 & 3.02 & $1.0 \cdot 10^{-4}$ & 5510 & 11 & 0.313 & $0.98 \cdot 10^{-6}$ & 50946 & 10 \\
\hline
\end{tabular}

The most important result of table 2 is the fact that the step size determination method predicts step sizes that result in actual root-mean-square errors for the propagation of the full signal which are very close to the target error. This good step size determination is obtained although $E(h)$ may depend on $h$ in a very ungraceful way, for instance as shown in fig. 1 which depicts $E(h)$ for case v. It is also very important that the time spent on the step size determination is only a small percentage of the total computation time, typically smaller than $20 \%$ (Two situations with very long step sizes and total computation times shorter than 1 second are exceptions).

\section{Conclusion}

We have proposed a new, simple and fast method for determination of the step size that results in a prescribed value of the error which is introduced when the split-step Fourier method is used to compute the propagation of optical signals through nonlinear fibres. It has been verified that the determined step size actually leads to an error which is very close to the prescribed value. Furthermore, the time spent on the step size determination is a small fraction of the total computation time, typically $20 \%$ or less. This possibility of a fast determination of the largest step size which is compatible with a given accuracy makes it possible to minimize the computation time. At the same time the method eliminates the risk of inaccurate results as a consequence of using too long steps. The method handles both single channel and WDM transmission. Furthermore, it is expected to be useable for other numerical techniques than the split-step Fourier method.

\section{References}

[1] Masashi Eguchi et al., "Analysis of a nonlinear Schrödinger equation with numerical techniques", J. Appl. Phys. 72, 3255-3257 (1992)

[2] Cristian Francia, "Constant step-size analysis in numerical simulation for correct four-wave-mixing power evaluation in optical fiber transmission systems", IEEE Photon. Technol. Lett. 11, 69-71 (1999) .

[3] G. Bosco et al., "Suppression of spurious tones induced by the split-step method in fiber systems simulation", IEEE Photon. Technol. Lett. $12,489-491(2000)$

[4] Govind P. Agrawal, Nonlinear Fiber Optics, second edition (Academic Press, 1995)

[5] William H. Press, Saul A. Teukolsky, William T. Vetterling, and Brian P. Flannery, Numerical Recipes in C, second edition (Cambridge University Press, 1992) 